\title{
First EMC3-Eirene simulations of the impact of the edge magnetic perturbations at ASDEX Upgrade compared with the experiment
}

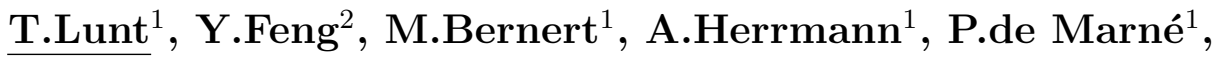 \\ R.McDermott $^{1}$, H.W.Müller ${ }^{1}$, S. Potzel $^{1}$, T.Pütterich ${ }^{1}$, \\ S.Rathgeber ${ }^{1}$, W.Suttrop ${ }^{1}$, E.Viezzer ${ }^{1}$, E.Wolfrum ${ }^{1}$, \\ M.Willensdorfer ${ }^{3}$ and the ASDEX Upgrade team ${ }^{1}$ \\ E-mail: tilmann.lunt@ipp.mpg.de \\ ${ }^{1}$ Max-Planck-Institut für Plasmaphysik, EURATOM Association, Boltzmannstr. 2, \\ 85748 Garching, Germany \\ ${ }^{2}$ Max-Planck-Institut für Plasmaphysik, EURATOM Association, Wendelsteinst. 1, \\ 17491 Greifswald, Germany \\ ${ }^{3}$ Institut für Angewandte Physik, Technische Universität Wien, Association \\ EURATOM-ÖAW, 1040 Vienna, Austria
}

\begin{abstract}
The EMC3-Eirene code package was applied for the first time to simulate the edge plasma in an ASDEX Upgrade discharge, in which the newly installed magnetic perturbation coils were used to mitigate Edge Localized Modes (ELMs). Two different points in time during this discharge were simulated, the ELM mitigated phase after turning-on of the magnetic perturbation coils and, as a reference, the ELMy H-mode phase before. The results were compared to the measurements of various edge and divertor diagnostics. Assuming the main chamber profiles to be shifted by $15 \mathrm{~mm}$ with respect to their calibrated positions, an agreement within a factor of 2 was found between the main chamber profiles outside the separatrix and those at the outer divertor target. The most important result is the observation of several maxima and minima in the particle flux and in particular in the power deposition pattern of both the simulation and the experiment for the case with magnetic perturbations, an effect also known as strike-point splitting.
\end{abstract}

\section{Introduction}

The high confinement regime in most tokamaks is accompanied by the appearance of so called edge localized modes (ELMs), quasi-periodic expulsions of particles and energy from the edge plasma to the surrounding plasma facing components (PFCs). Due to the limited capability of the PFCs to withstand the enormous heat loads expected in particular in large devices, ELMs have to be controlled or even suppressed in ITER. An important success to provide such a control tool was reported by several devices (COMPASS [1], DIII-D [2, 3, 4, 5], JET [6, 7], .. ) where magnetic perturbations (MPs) in the plasma edge were applied. Presently, a new set of 24 in-vessel MP coils is being 
installed at ASDEX Upgrade (AUG), of which eight coils started operating in December 2010. Initial experiments show that these coils also reduce significantly the ELMs at AUG $[8,9]$. In order to make predictions for ITER, where the application of MP coils is also planned, a validated theoretical model to describe the edge plasma transport is needed. Since the MP coils break the toroidal symmetry of the tokamak, such a model has to take into account the full 3D geometry including the stochastic fields induced by the coils. Recently, the Edge Monte Carlo 3D - Eirene code package (EMC3-Eirene) was implemented successfully at AUG and validated for limiter [10] and divertor discharges $[11,12]$. In this article we will report on first simulations with EMC3-Eirene of the effect of the MP coils and compare them to the measurements of various edge and divertor diagnostics.

\section{Experiment}

Since the installation of the MP coils in 2010 many discharges were performed to investigate the impact of the perturbation fields on the edge plasma stability, the particle and energy fluxes to the targets and the impurity content in the confinement region under different experimental conditions. An overview over these experimental findings can be found in Refs. [8, 9]. In this article we concentrate on a single configuration and document the results as much in detail as possible. Besides relevant discharge parameters the most important criterion for the selection of an appropriate discharge was the availability of experimental data from various edge and divertor diagnostics, which were found to be most comprehensive in the case of the ELM-mitigated AUG Hmode discharge 26081. Figure 1 shows the time traces of selected discharge parameters. During the flattop phase $(1-7 \mathrm{~s})$ the plasma current remains constant at $I_{p}=-800 \mathrm{kA}$ as seen in box (a), as well as the particle and power (boxes (c) and (d)) input. From $2-5.3 \mathrm{~s}$ a current of $I_{M P}= \pm 0.9 \mathrm{kA} \times 5$ turns $= \pm 4.5 \mathrm{kAt}$ is driven through the two sets of perturbation coils, which are connected in an odd parity $n=2$ configuration. An important observation is that neither the line averaged density, $\bar{n}_{e}$, nor the stored energy, $W_{M H D}$, (black and red curves in box (b)) are reduced by the perturbation fields. The first actually shows a slight increase rather than a 'density pump-out' as observed in other devices $[5,6,13]$. Although the plasma performance is not influenced by the perturbation fields the edge localized modes (ELMs) are effectively suppressed as seen in the data from the 1D infrared camera (box (e) showing color coded the power flux versus the divertor coordinate $s$ ). While ELMs deposit large amounts of energy in frequent eruptions on a short time scale before the turning-on of the coils (scattered signals in box (d) from 1-2.5 s) the power deposition becomes much smoother about half a second after (i.e. from about $2.5 \mathrm{~s}$ on). In addition, the profile shows several maxima and minima during the phase 3-5 s, an effect also known as 'strike-point splitting' $[14,4,5]$. From now on we focus on the time point $t=4.9 \mathrm{~s}$ indicated by the red line in Fig. 1 , which we will also refer to as the 'MP case', as well as the reference 'non-MP case' 
at time $t=1.9 \mathrm{~s}$ (blue vertical line) just before the turning-on of the coils. For both cases the external discharge conditions are about the same, the toroidal magnetic field is $B_{t}=-2.5 \mathrm{~T}$ and the safety factor at $95 \%$ of the poloidal flux is $q_{95}=-5.7$. $P_{N I}=7.3 \mathrm{MW}$ of power and $\Phi_{N I}=9.5 \cdot 10^{20} \mathrm{~s}^{-1}$ deuterium atoms are deposited in the core region by neutral beam injection (NBI). In addition to the NBI the plasma is heated by $P_{E C R H}=1.6 \mathrm{MW}$ of ECRH and $P_{O H}=0.16 \mathrm{MW}$ of ohmic power. Tomographic reconstruction of bolometry shows that the major part of the total input power $P_{t o t}=\Phi_{N I}+P_{E C R H}+P_{O H}=9 \mathrm{MW}$ leaves the plasma in form of electromagnetic radiation, originating in particular from the SOL near the inner divertor target. The radiative power loss $P_{\text {rad }}$ is at least $5 \mathrm{MW}$ or $56 \%$, however, since two channels of the bolometer were saturated this value might still be underestimated. The power fluxes to the inner and outer targets were measured by the 1D infrared camera to be $P_{i t}=0.6$ $\mathrm{MW}$ and $P_{o t}=2 \mathrm{MW}$, respectively. Given that neither main chamber PFCs nor the divertor roof baffle are expected to receive significant amounts of power, the $1.4 \mathrm{MW}$ remaining to fulfill the power balance are attributed to the underestimation of $P_{\text {rad }}$, to $P_{i t}+P_{o t}$ or to both of them.

\section{Code and computational grid}

EMC3-Eirene is applied to simulate the edge plasma, the neutral particle and the impurity transport in full 3D geometry. Originally it was developed for the intrinsically three dimensional W7-AS [15] stellarator. During the last few years, however, the code also found application in the tokamak community in particular since magnetic perturbation coils are used, which break the toroidal symmetry of the tokamak. The working principle as well as the full set of equations solved by EMC3-Eirene are described in detail in Ref. [16]. Here, we only want to summarize that EMC3 solves Braginskii's equations and is self-consistently coupled to Eirene, which solves the kinetic equation for the neutrals. Whereas the parallel transport is described purely neoclassical in EMC3, anomalous cross-field transport coefficients $D, \eta$ and $\chi$ are assumed as free parameters in the code. Although a Monte Carlo principle is applied to solve the equations numerically, the code assumes a fluid model to describe the plasma including all the approximations of the fluid picture. Despite the completely different numerical method and the different type of computational grid benchmarks against the 2D fluid codes SOLPS and EDGE2D-Eirene have recently shown reasonably good agreement for the axisymmetric case $[10,17,18]$. It has to be mentioned, however, that the present version of EMC3 includes a less comprehensive set of atomic processes (volumic recombination excluded for example) and drifts and are not taken into account.

In order to simulate a particular plasma experiment with EMC3-Eirene it is necessary to describe the geometry of both the plasma facing components (PFCs) and the magnetic field. While the former is relatively simple, since for this first application we only take into account the toroidally symmetric components, the latter is clearly non-trivial. Here 
we assume the 'vacuum approach', meaning that the magnetic field is given by

$$
\begin{aligned}
& \vec{B}=\vec{B}_{E Q}+\vec{B}_{M P}, \text { with } \\
& \vec{B}_{E Q}=-\frac{1}{R} \frac{\partial \Psi}{\partial z} \vec{u}_{R}+\frac{1}{R} \frac{\partial \Psi}{\partial R} \vec{u}_{z}+\frac{\mu_{0} f}{R} \vec{u}_{\phi}
\end{aligned}
$$

where $\vec{u}_{R}, \vec{u}_{z}$ and $\vec{u}_{\phi}$ are the unit vectors in radial, axial and toroidal directions and where the poloidal magnetic flux $\Psi$ and the current flux function $f$ are computed by the 2D equilibrium code Cliste [19]. The magnetic field $B_{M P}$ caused by the perturbation coils is obtained by integration of the Biot-Savart formula approximating the MP coils by current filaments surrounded by empty space. Any response of the currents in the plasma to the perturbation coils is neglected so far.

The magnetic geometry is read in by EMC3 in the form of a computational grid, which is not only needed to count weighted Monte Carlo particles within finite size grid cells and like this to calculate local plasma parameters, but is also used for pre-calculating the field on a large number of interpolation points, enabling a fast and accurate tracing of field lines (cf. the 'reversible field line mapping' technique described in Ref. [15]). The generalization of the field line reconstruction method for block-structured grids, which is required for poloidal divertor configurations, has been presented by Frerichs et al. [20]. Formally the grid is divided into $N_{z}$ zones labeled with the 'zone' index $i z=0 \ldots N_{z}-1$. Each zone constitutes a structured cubic grid, meaning that for every 'radial', 'poloidal' and 'toroidal' index $i r=0 \ldots N_{r}(i z)-1, i p=0 \ldots N_{p}(i z)-1$ and $i t=0 \ldots N_{t}(i z)-1$ a grid vertex

$$
\vec{G}_{i r, i p, i t}^{i z}
$$

exists and that cells with neighboring indices are also neighbors in real space. For a given zone $i z$ we will refer to the subset of grid points with a constant radial, poloidal or toroidal index as a radial, poloidal or toroidal surface, respectively. A precondition for the code is also that the toroidal angle $\phi$ is constant on toroidal planes.

Since the perturbation fields have an $n=2$ symmetry the toroidal extension of the grid was chosen as $180^{\circ}$. The bilinear field-line interpolation method used in EMC3 requires convex cells [15]. Because of finite-shear effects this is possible only for field lines of limited lengths. For this reason, the $180^{\circ}$ toroidal interval is divided into 8 'sectors' with three zones, core, scrape-off layer (SOL) and private flux region (PFR) each, yielding a total of 24 zones. A poloidal cross section of the grid covering an interval of about $\Psi_{N}=0.85 \ldots 1.09$ of the normalized poloidal flux is shown in Fig. 2. It is underlayed by a Poincaré plot (black dots) which shows clearly the appearance of several island chains in the core region, at least when assuming the aforementioned vacuum approach.

\subsection{Grid optimization}

For the simulation of the non-axisymmetric situation with MPs two important measures were taken to optimize the grid: First, one has to avoid the introduction of toroidal 
asymmetries at the grid-target interface due to the finite resolution of the grid ('Moiré effects'). For this reason the poloidal spacing of the grid is chosen such that grid points at the grid-target interface lie exactly on the target. Second, another measure to optimize the grid is to choose a so called 'quasi flux surface' (QFS) for the innermost radial surface of the plasma domain to isolate the parallel transport. A QFS is a surface in 3D space on which the radial component of the parallel transport is much smaller than the crossfield transport. Only on these surfaces can we avoid formulating boundary conditions for the parallel transport. The construction of QFSs is carried out in the following way: A field line starting from a point $\left(R_{0}, z_{0}, \phi=0\right)$ is traced a few (typically 30) toroidal field periods $\Delta \phi$. The points $\left(R_{i}, z_{i}, \phi=i \Delta \phi\right)$ that lie on that field line at $i$ field periods are projected into the $\phi=0$ plane and connected by a set of $\left(\mathfrak{R}_{j}, \mathfrak{z}_{j}\right)$ points on splines. These points are in turn followed one field period to form a curve $\left(\mathfrak{R}_{j}^{\prime}, \mathfrak{z}_{j}^{\prime}\right)$. Now $R_{0}, z_{0}$ are varied such that the area between the two curves $\left(\mathfrak{R}_{j}, \mathfrak{z}_{j}\right)$ and $\left(\mathfrak{R}_{j}^{\prime}, \mathfrak{z}_{j}^{\prime}\right)$, the 'area-mismatch', becomes minimal. Due to the presence of resonances in the MP fields the area mismatch is a strong function of the radius. QFSs with satisfactory quality (that permit only a negligible number of Monte Carlo particle to escape) can always be found with this method.

\section{Results}

Assuming the experimental boundary conditions described in Sec. 2 and constructing a computational grid according to the methods described in previous Sec. 3 plasma parameters as well es neutral particle densities were computed by means of EMC3Eirene. Since impurities were not included in the simulation so far, we subtract the measured radiation losses from the input power. As discussed in Sec. 2 we presume $P_{\text {rad }}$ to be slightly higher than actually measured by bolometry and assume an input power flux of $P_{i n}=3.3 \mathrm{MW}$ through the innermost radial surface of the computational domain, which is equally distributed between electrons and ions. 3D representations of electron density $n_{e}$, Mach number $M=u / c_{s}$ (streaming velocity $u$ normalized to the speed of sound $c_{s}$ ) and electron temperature $T_{e}$ are shown in Fig. 3. The green and red curves represent the perturbation coils with positive and negative currents respectively. The helical structures provoked by these coils are clearly visible in particular in the field for $M$ (Fig. 3 middle). A poloidal cross-section of $M$ in the divertor at a toroidal angle $\Phi=0^{\circ}$ is also shown in Fig. 4, where the case with and without MP fields is compared. Due to the strongly modified connection lengths with the inner and outer targets regions with significantly larger fluxes and alternating sign (red and blue represent positive and negative streaming directions, respectively) are found for the case with MP fields. It is not surprising that such a strong change of the transport in the edge plasma potentially influences also its stability. The physical mechanism of the ELM mitigation and the conditions under which it is achieved cannot be identified so far and have to be investigated in a more systematic study.

In order to obtain the main chamber $n_{e}, T_{e}$ and $T_{i}$ profiles represented by the black 
curves in Figs. 5 and 7 constant anomalous particle and heat diffusion coefficients of $D_{\perp}=0.35 \mathrm{~m}^{2} / \mathrm{s}$ and $\chi_{\perp}=2.5 \mathrm{~m}^{2} / \mathrm{s}$ and a separatrix density of $n_{e, s e p}=2.0 \cdot 10^{19}$ $\mathrm{m}^{-3}$ were assumed. Fig. 5 corresponds to the MP case, while Fig. 7 shows the non-MP situation for comparison. For the vacuum approach with constant transport coefficients, the MP fields provoke a strong additional radial transport (the parallel transport has a radial component, when the MP fields are turned on) and, therefore, a slight flattening of the simulated density profile and a strong reduction of the temperature at the innermost boundary of the computational domain. The fact that no density pump-out and no reduction of the stored energy in the core is observed in the experiment, is a strong indication for the insufficiency of the vacuum approach and the much lower stochasticity of the fields in the experiment. It might be, however, that the anomalous transport and that caused by the stochastic fields are affected at the same time and that these two effects exactly cancel out, although this would be a rather unlikely coincidence. The consistent implementation of screening effects as proposed recently by several authors , e.g. $[1,21]$, therefore is a pending task for the near future. In any case it has to be mentioned that the edge transport code EMC3 does not include the main physics of the core transport (transport barrier, inward pinch, kinetic effects, ...) and so in the following we focus mostly on the profiles outside the separatrix and downstream in the divertor.

The simulated main chamber profiles are compared to the measurements of the lithium beam and charge exchange spectroscopy, while the divertor Langmuir probes and the 1D infra-red camera provide experimental data for the divertor (Figs. 6 and 8). The simulated main chamber $T_{e}$ profile is also shown together with the one measured by the electron cyclotron emission (ECE) diagnostics, however, a comparison is not possible since the two contain complementary information. The ECE data is reliable only inside the separatrix, while EMC3 describes the plasma transport best in the SOL. In order to reduce the statistic errors of the experimental data all profiles were averaged over a time interval of $100 \mathrm{~ms}$. During the ELMy non-MP phase we furthermore distinguish the average profiles (red curves) from profiles averaged over the inter-ELM phases (magenta curves) only. Due to the finite accuracy of the equilibrium reconstruction, the measured radial profiles seem to be shifted with respect to the separatrix position. The separatrix is assumed to be at the position, where the slope of the density profile in the non-MP phase (Fig. 7 top) strongly changes due to the presence of an edge transport barrier. The measured and simulated $n_{e}$ profiles then agree within the error bars at least in the $\operatorname{SOL}\left(R-R_{\text {sep }}=0 \ldots 25 \mathrm{~mm}\right)$. Consistently, the same shift of $\Delta R=-15 \mathrm{~mm}$ is applied to all other profiles.

Figures 6 and 8 finally show the simulated particle and power fluxes in the outer divertor compared to those measured by the divertor Langmuir probes and the 1D infra-red camera. Again the MP case (Fig. 6 bottom) is compared to the case without MPs (Fig. 8). First of all we note that the absolute value for the ion saturation current $j_{\text {sat }}$ agrees within a factor of two and that the experimental profiles are reproduced relatively well by the simulation, at least in the wing, i.e. for $s=1.12 \ldots 1.27 \mathrm{~m}$. There are, however, 
systematic discrepancies in the near strike-point region, i.e. for $s=1.12 \ldots 1.27 \mathrm{~m}$ which indicate the onset of partial detachment of the outer divertor for the non-MP phase. Since we did not take into account the impurity transport so far, it is not surprising that we do not capture this effect in the code.

Due to the exclusion of the impurity transport in the simulation we cannot expect a better agreement than the $40 \%$ difference between the measured and simulated absolute values for the power flux (note the different scales for the two profiles in Figs. 6 and 8). Apparently part of the power is reaching the outer target in form of radiation (produced in a small region in front of the strike zone), which could explain the approximately homogeneous offset of $P=0.5 \mathrm{MW} / \mathrm{m}^{2}$ in the experimental power deposition profile. This effect will be investigated quantitatively in the future simulating the impurity line radiation in the divertor.

Independently, an important result is the observation of the strike-point splitting, i.e. the appearance of several maxima and minima in the vicinity of the nominal strike point (solid black vertical line) caused by the MP fields. Due to the limited spatial resolution this effect is only marginally observable by the Langmuir probes and only when comparing the MP and the non-MP cases around $s=1.2 \mathrm{~m}$. In contrast, the power flux pattern measured by the 1D IR camera shows 4 clearly distinguishable maxima. While the ratio of the heights of the peaks is in good agreement with the simulation a shift in the spatial position is found with respect to the measurement, which is most likely due to inaccuracies in the equilibrium reconstruction and/or the target geometry. Possibly local currents flowing between the inner and outer targets have to be taken into account. The characteristic shape of the simulated power deposition pattern is nevertheless very similar to the experimental one and in addition to that the maxima and minima disappear in both the simulation and experimentally as soon as the MP coils are turned off (cf. Fig. 8). This confirms that the physical and geometrical effects leading to the strike point splitting are actually captured by EMC3-Eirene even when neglecting screening effects for the MP fields in the confinement region.

A similar study as the one described here for AUG was also carried out by Frerichs et al. [4] and Schmitz et al. [5] for DIII-D. EMC3-Eirene was also used there to compute particle and power deposition profiles which were compared to the experimental ones. While the strike point splitting was observed in the $\mathrm{D}_{\alpha}$ signal (although less pronounced), no splitting was found for the experimental power flux. It has to be pointed out, however, that the pedestal electron collisionality in [4] and [5] is very low. Given that a second peak in the power deposition profile could be resolved at higher collisionalities [14] kinetic effects might have to be taken into account in the modeling to explain this discrepancy. A gradual disappearance of the splitting with decreasing collisionality was also observed by Jakubowski et al. [22].

Other authors addressed the strike point splitting from a purely geometrical approach by computing connection lengths. While some of them $[13,22,23,24]$ also compare their results the experimental data, others [25, 26] follow a purely theoretical approach. 


\section{Summary and outlook}

For the first time, non-axisymmetric divertor plasmas in AUG with the presence of magnetic perturbations were simulated using the EMC3-Eirene code package. Two different time points during one single discharge were compared, the ELMy 'nonMP phase' before the turning-on of the newly installed perturbation coils in ASDEX Upgrade and the ELM mitigated 'MP phase' after. The results were compared to the measurements of various edge and divertor diagnostics. Assuming the main chamber profiles to be shifted by $15 \mathrm{~mm}$ with respect to their calibrated positions, an agreement within a factor of 2 was found between the main chamber profiles outside the separatrix and those at the outer divertor target. The most important result is the observation of several maxima and minima in the experimental and simulated power deposition profiles in the outer divertor, an effect also known as strike-point splitting. This effect vanishes in both experiment and simulation, when the MP coils are turned off, which confirms that the physical mechanisms and the geometry leading to the splitting are actually captured by EMC3-Eirene.

It was furthermore found that the MP fields have a strong impact on the transport in the plasma edge, where large helical flows with alternating sign appear. It is not surprising that this effect potentially can influence the stability of the edge plasma, however, the question of how and under which conditions the ELM mitigation mechanism actually

works remains open. A more refined and systematic study of the influence of the MP field spectrum as well as a comparison of the conditions where ELM mitigation is achieved and where not is planed for the near future.

The fact that no 'density pump-out' and no reduction of the stored energy was observed in the experiment is a strong indication for the invalidity of the vacuum approach and for the overestimation of the stochastic effects on the core transport implying the relevance of the response of plasma currents to the perturbation fields. The consistent implementation of these screening effects is also planned as one of the next steps. The good agreement of the splitting of the power load profiles between the code and the 1D IR camera on the other hand indicates that the SOL power channels most likely originate from a near-separatrix region outside the effective screening domain.

\section{References}

[1] Cahyna P. el al. 2011 J. Nucl. Mater. 415 S927-S931

[2] Evans T. et al. 2004 Phys. Rev. Lett. 92235003

[3] Evans T. et al. 2008 Nucl. Fusion 48024002

[4] Frerichs H. et al. 2010 Nucl. Fusion 50034004

[5] Schmitz O. et al. 2008 Plasma Phys. Control. Fusion 50124029

[6] Liang Y. et al. 2007 Phys. Rev. Lett. 98265004

[7] Liang Y. et al. 2007 Plasma Phys. Control. Fusion 49 B581

[8] Suttrop W. et al. 2011 Phys. Rev. Lett. 106225004

[9] Suttrop W. et al. 2011 Studies of ELM-mitigation with new active in-vessel saddle coils in ASDEX Upgrade accepted for publication Plasma Phys. Control. Fusion 
First EMC3-Eirene simulations of the impact of the edge MPs at AUG compared with the experiment9

[10] Lunt T. el al. 2009 Proc. 36 ${ }^{\text {th }}$ EPS Conference on Plasma Phys., Sofia, Bulgaria, P-1.154, June 29-July 3

[11] Lunt T. el al. 2011 J. Nucl. Mater. 415 S505-S508

[12] Lunt T. el al. 2011 EMC3-Eirene simulations of the spatial dependence of the tungsten divertor retention in ASDEX Upgrade accepted for publication in Plasma Phys. Control. Fusion

[13] Nardon E. et al. 2009 Plasma Phys. Control. Fusion 51124010

[14] Evans T. et al. 2005 J. of Phys. Conference Ser. 7 174-190

[15] Feng Y.et al. 2002 Plasma Phys. Control. Fusion 44611

[16] Feng Y. et al. 2004 Contrib. Plasma Phys. 44, No. 1-3, 57-69

[17] Harting D. el al. 2011 J. Nucl. Mater. 415 S540-S544

[18] Feng Y. el al. 2011 EMC3-Eirene/SOLPS4.3 comparison for ITER Proc. $38^{\text {th }}$ EPS Conference on Plasma Phys., Strasbourg, France, P-1.071, June 27-July 1

[19] Mc Carthy P.J. 1999 Phys. of Plasmas 63554

[20] Frerichs H. et al. 2010 Comput. Phys. Commun. 181 61-70

[21] Yu Q. and Günter S. 2009 Nucl. Fusion 49062011

[22] Jakubowski M.W. et al. 2009 Nucl. Fusion 49095013

[23] Harting D. et al. 2011 Strike point splitting in the heat and particle flux profiles compared to the edge magnetic topology in a n=2 resonant magnetic perturbation field at JET submitted to Nucl. Fusion

[24] Fuchs J.C. 2011 Influence of non-axisymmetric magnetic perturbations on the equilibrium reconstruction at ASDEX Upgrade Proc. $38^{\text {th }}$ EPS Conference on Plasma Phys., Strasbourg, France, P-1.090, June 27-July 1

[25] Wingen A. et al. 2009 Nucl. Fusion 49055027

[26] Wingen A. et al. 2009 Phys. of Plasmas 16042504 
First EMC3-Eirene simulations of the impact of the edge MPs at AUG compared with the experiment 10

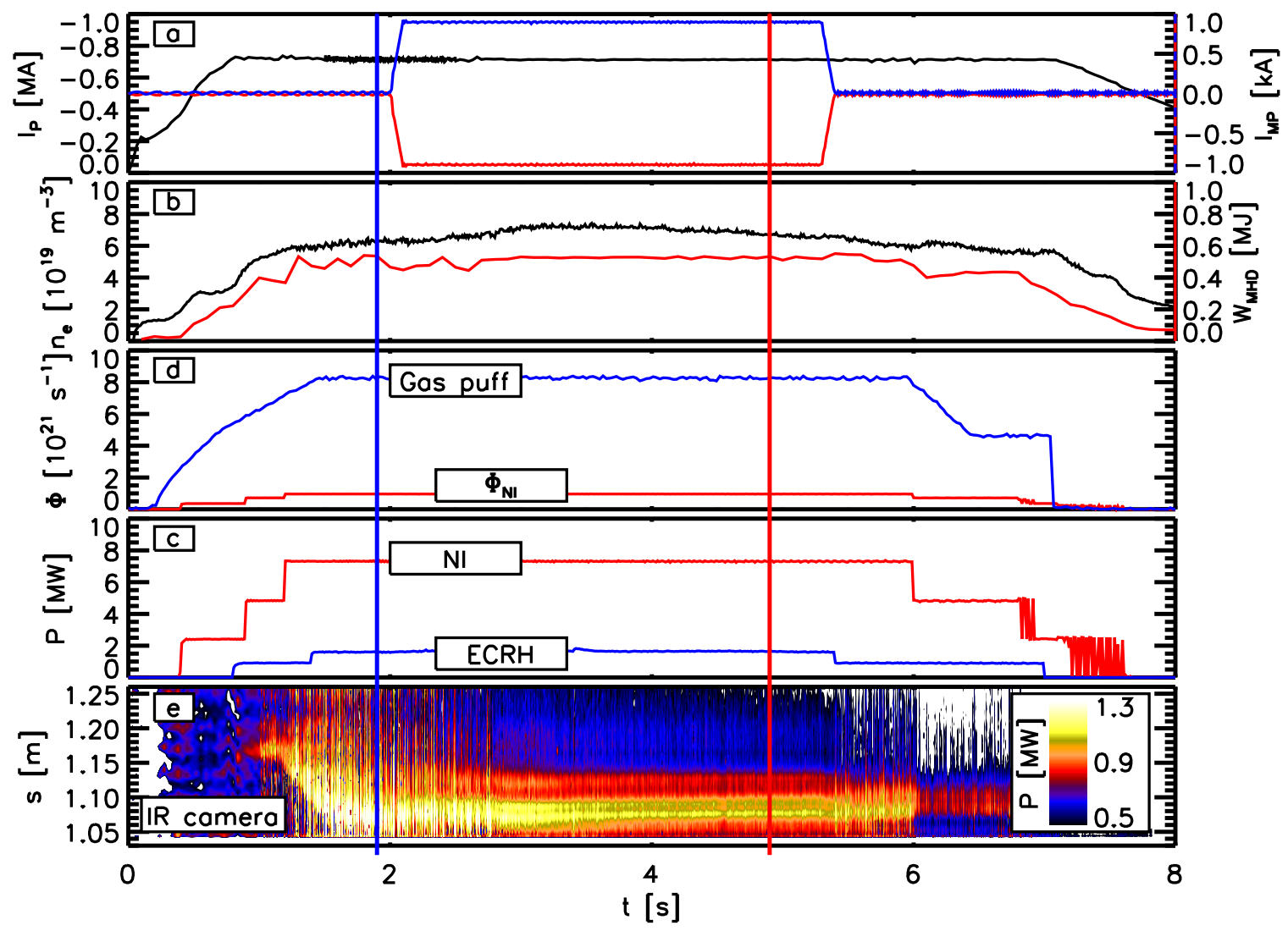

Figure 1. Time traces of selected discharge parameters of AUG pulse 26081. 


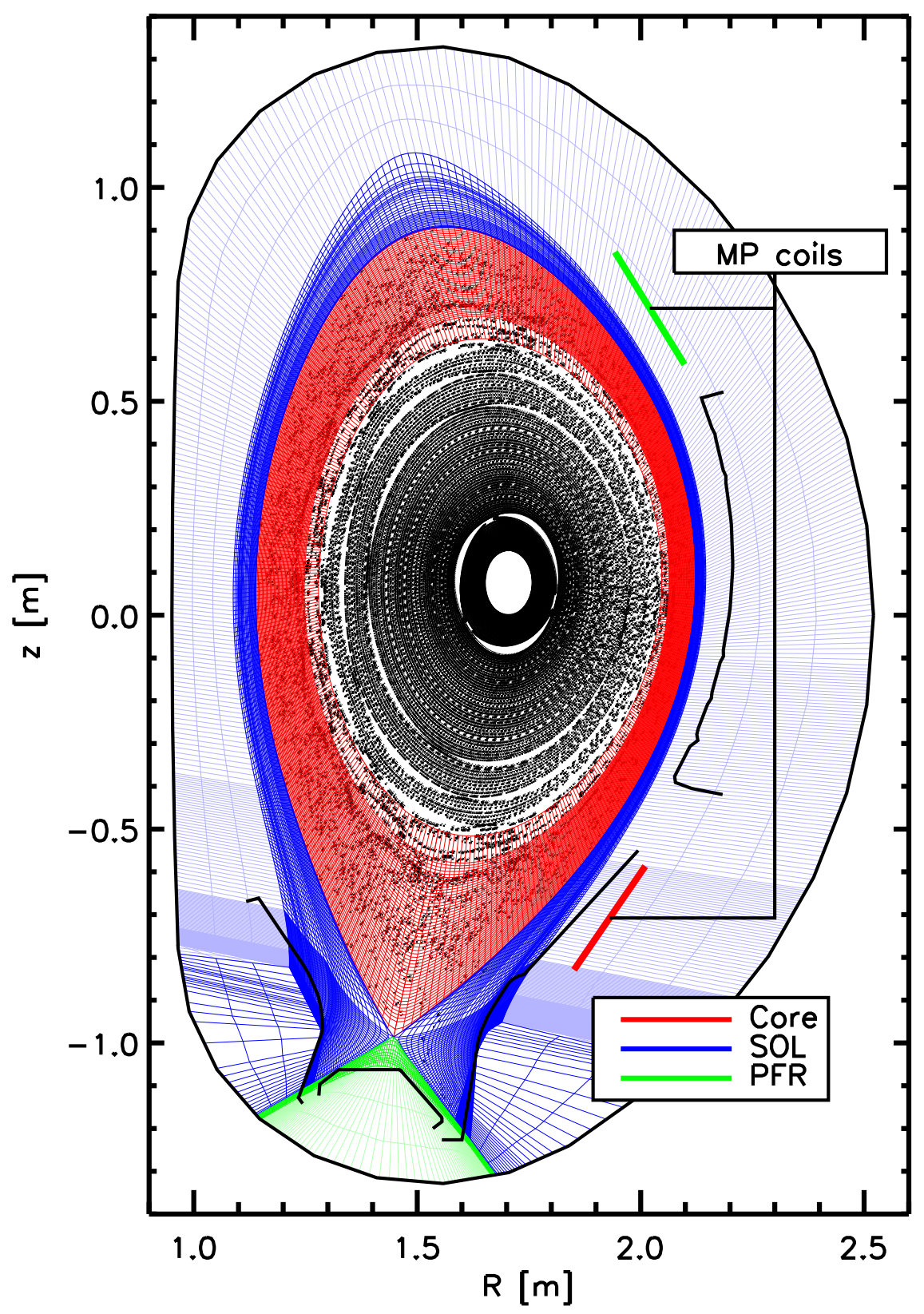

Figure 2. Poincaré plot for AUG discharge 26081 at $4.9 \mathrm{~s}$ with $4.5 \mathrm{kAt}$ current in the MP coils assumig the vacuum approach. The computational grid for EMC3-Eirene is overplotted. For each of the 8 segments (together $180^{\circ}$ of the toroidal circumference) it is divided into three zones, 'core' (red), 'SOL' (blue) and private flux region ('PFR', green). 
First EMC3-Eirene simulations of the impact of the edge MPs at AUG compared with the experiment 12

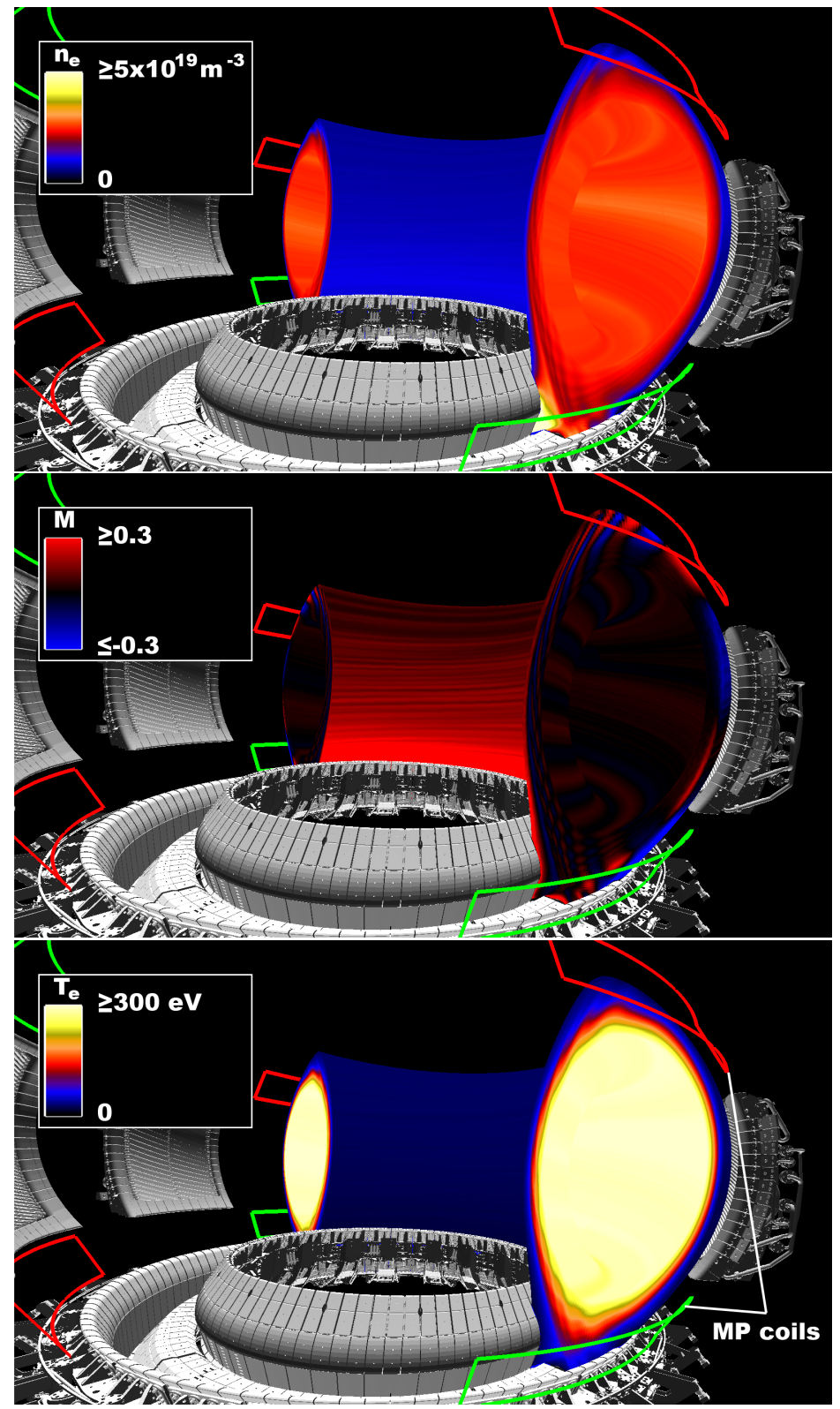

Figure 3. 3D plots of $n_{e}, M$ and $T_{e}$ computed by means of EMC3-Eirene for AUG discharge 26081 at $4.9 \mathrm{~s}$. The green and red loops represent the magnetic perturbation coils $\left(\left|I_{M P}\right|=4.5 \mathrm{kAt}\right)$ with positive and negative coil currents respectively. 
First EMC3-Eirene simulations of the impact of the edge MPs at AUG compared with the experiment13
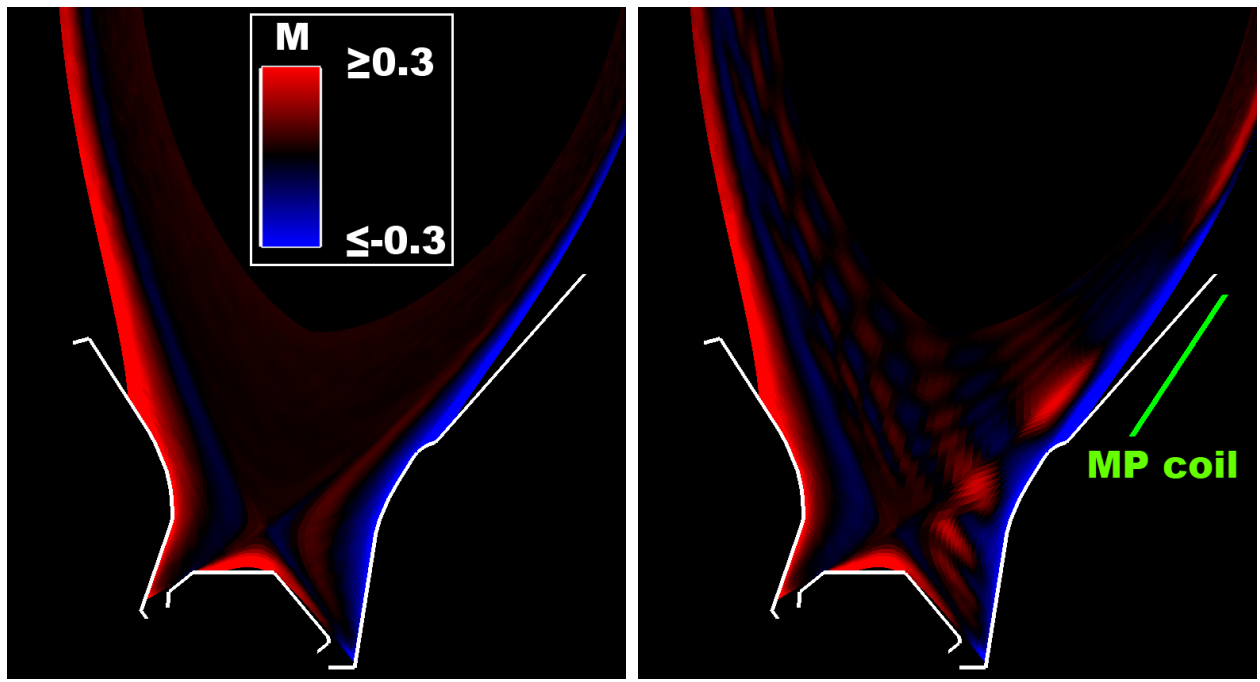

Figure 4. Cross section of the Mach number (cf. Fig. 3 middle) at the toroidal position $\Phi=0^{\circ}$. The left figure shows the case without and the right figure the case with MP fields. With MP fields strong helical structures form with alternating flux direction. 


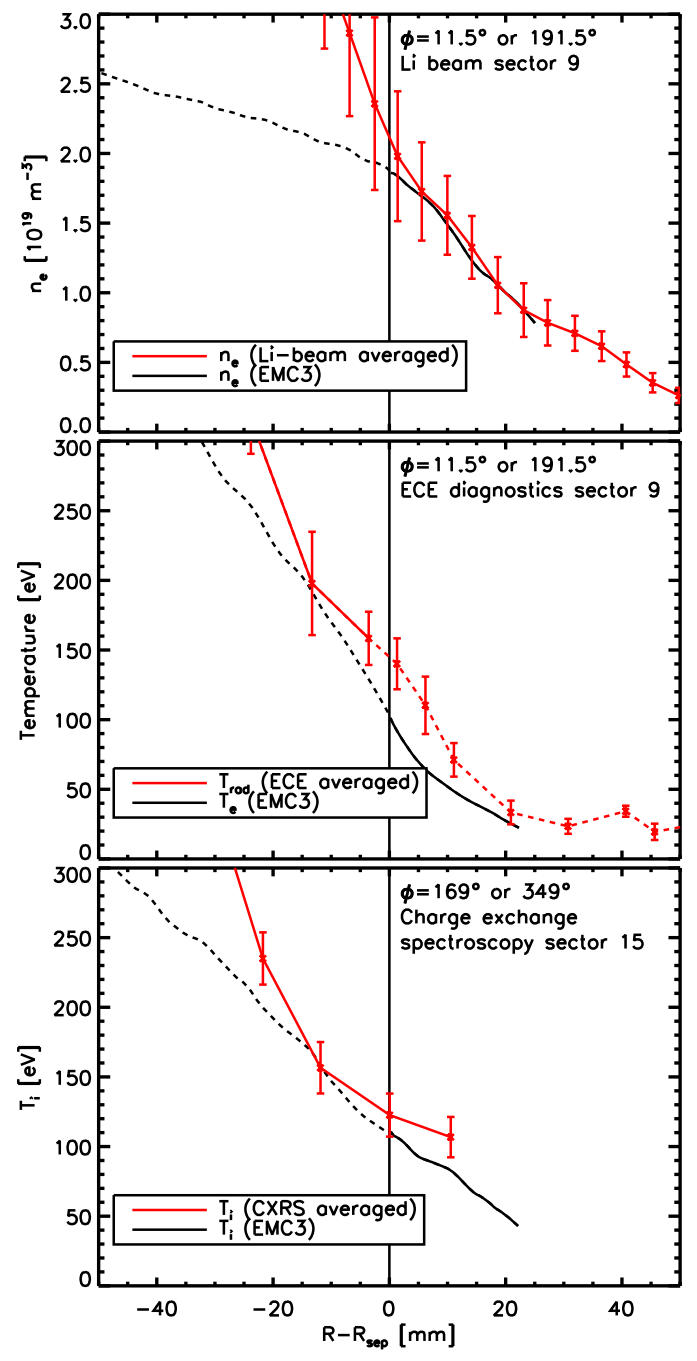

Figure 5. $n_{e}$ (top), $T_{e}$ (middle) and $T_{i}$ (bottom) profiles in the main chamber for AUG discharge 26081 at $4.9 \mathrm{~s}$ as measured by the Li-beam, the ECE diagnostics and charge exchange spectroscopy respectively (red data points). In order to correct an inaccuracy of the equilibrium reconstruction, all curves were shifted by $\Delta R=-15$ $\mathrm{mm}$. The black curves show the profiles predicted by the EMC3Eirene simulation.

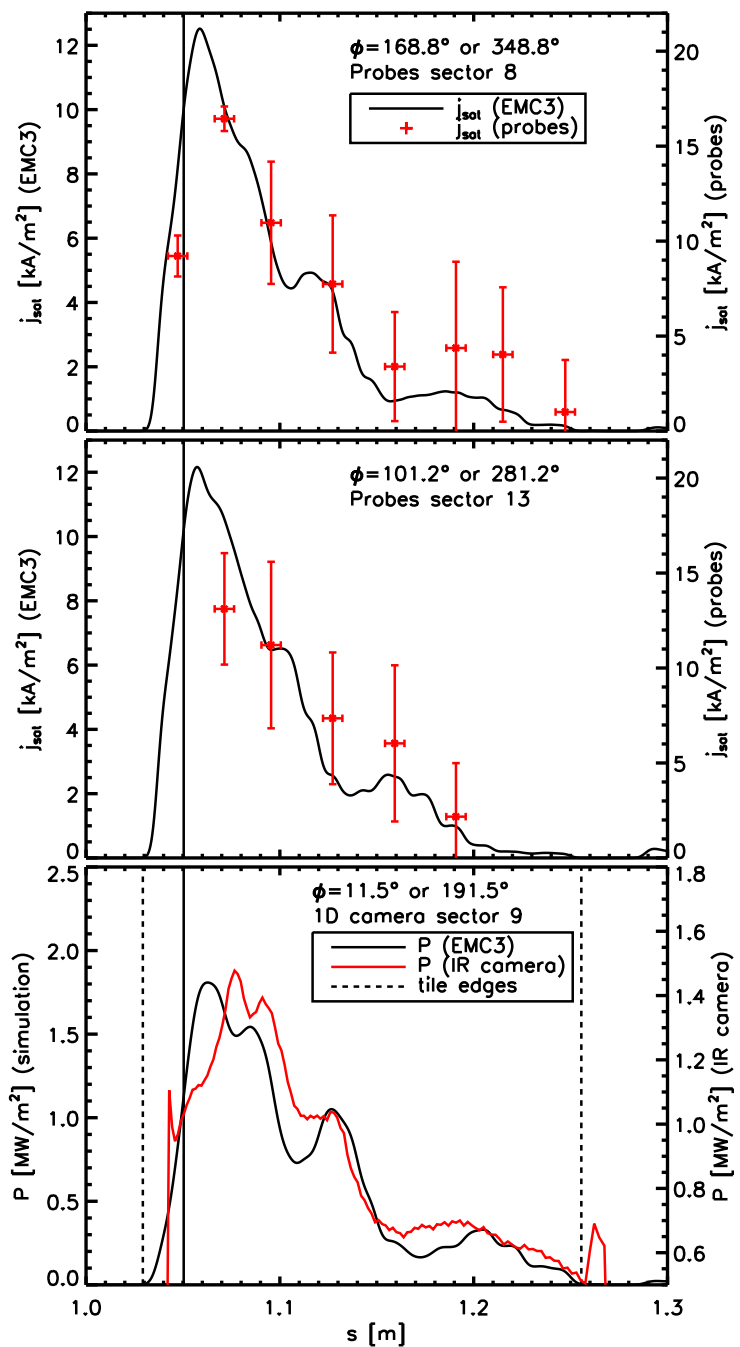

Figure 6. Divertor particle and power fluxes measured (red) and simulated by means of EMC3 (black) at the toroidal positions of the diagnostics. Note the different scales for the simulated (left) and experimental (right) data. 


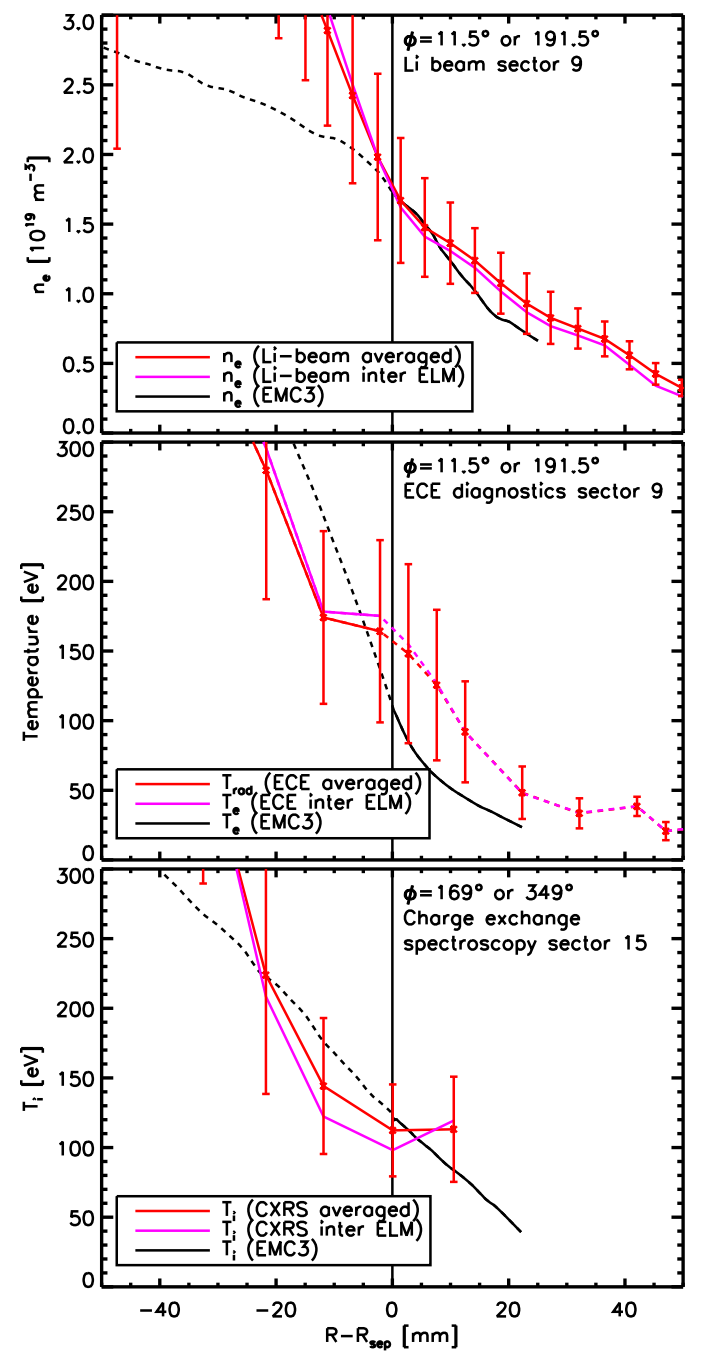

Figure 7. Same as Fig. 5 but for $t=1.9 \mathrm{~s}$ without magnetic perturbations. The same shift of $\Delta R=-15 \mathrm{~mm}$ was applied.

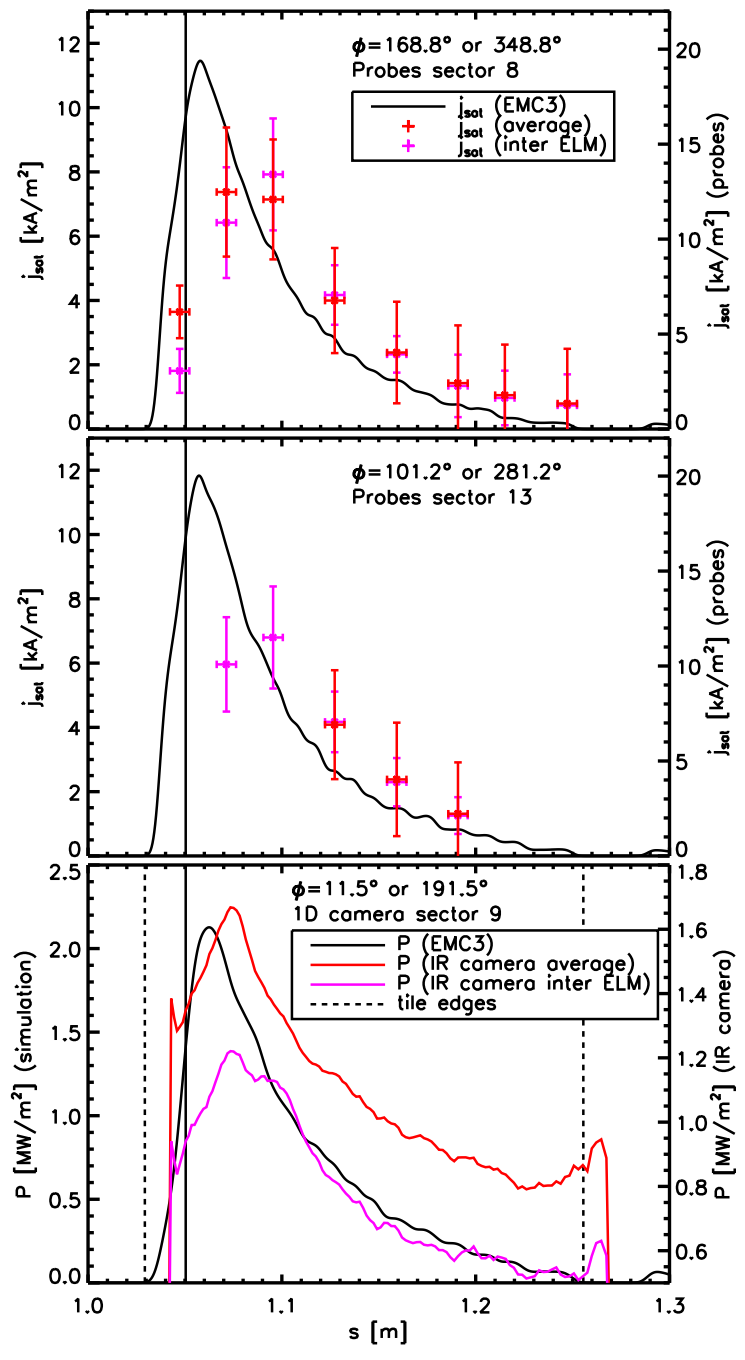

Figure 8. Same as Fig. 6 but for $t=1.9 \mathrm{~s}$ without magnetic perturbations. 